\title{
Comparing pain relief and functional improvement between methylprednisolone and dexamethasone lumbosacral transforaminal epidural steroid injections: a self-controlled study
}

\author{
Nicholas K. Donohue ${ }^{1}$, Sergey S. Tarima ${ }^{2}$, Matthew J. Durand ${ }^{1,3}$, and Hong $\mathrm{Wu}^{1,4}$ \\ 'Department of Physical Medicine \& Rehabilitation, Medical College of Wisconsin, Milwaukee, WI, USA \\ ${ }^{2}$ Division of Biostatistics, Medical College of Wisconsin, Milwaukee, WI, USA \\ ${ }^{3}$ Cardiovascular Center, Medical College of Wisconsin, Milwaukee, WI, USA \\ ${ }^{4}$ Department of Anesthesiology, Medical College of Wisconsin, Milwaukee, WI, USA
}

Received February 7, 2020

Revised March 5, 2020

Accepted March 6, 2020

\section{Correspondence}

Nicholas K. Donohue

Department of Physical Medicine \&

Rehabilitation, Medical College of

Wisconsin, 8701 Watertown Plank Road,

Milwaukee, WI 53226, USA

Tel: +1-414-805-7342

Fax: +1-414-805-7919

E-mail: nkdonohue@mcw.edu
Background: Previous studies have shown varying results between lumbosacral transforaminal epidural steroid injections (TFESIs) performed with particulate versus non-particulate corticosteroids. The purpose of this study was to investigate the difference in pain relief and functional improvement between particulate and nonparticulate lumbosacral TFESIs in patients who had undergone both injections, sequentially.

Methods: This was a self-controlled, retrospective study of 20 patients who underwent both a methylprednisolone and a dexamethasone TFESI to the same vertebral level and side. Primary outcomes included pain relief according to the visual analogue scale (VAS) and functional improvement determined by a yes/no answer to questions regarding mobility and the activities of daily living. Post-injection data was recorded at 2, 3, and 6 months.

Results: A decrease in VAS scores of $-3.4 \pm 3.0$ (mean \pm standard deviation), -3.1 \pm 3.1 , and $-2.8 \pm 3.4$ was seen for the methylprednisolone group at 2,3 , and 6 months, respectively. Similar decreases of $-3.9 \pm 3.5,-3.4 \pm 2.8$, and $-2.3 \pm 3.4$ were seen in the dexamethasone group. There was no significant difference in pain relief at any point between the two medications. The percentage of subjects who reported improved function at 2, 3, and 6 months was $65 \%, 51 \%$, and $41 \%$, respectively, for the methylprednisolone group and $75 \%, 53 \%$, and $42 \%$ for the dexamethasone group.

Conclusions: These findings support the use of non-particulate corticosteroids for lumbosacral TFESIs in the context of documented safety concerns with particulate corticosteroids.

Key Words: Adrenal Cortex Hormones; Dexamethasone; Injections, Epidural; Low Back Pain; Lumbosacral Region; Methylprednisolone; Pain Management; Radiculopathy; Steroids. (a) This is an open-access article distributed under the terms of the Creative Commons Attribution Non-Commercial License (http://creativecommons.org/licenses/by-nc/4.0/), which permits unrestricted non-commercial use, distribution, and reproduction in any medium, provided the original work is properly cited.

(c) The Korean Pain Society, 2020
Author contributions: Nicholas K. Donohue: Writing/manuscript preparation; Sergey S. Tarima: Formal analysis; Matthew J. Durand: Project administration; Hong Wu: Supervision. 


\section{INTRODUCTION}

Transforaminal epidural steroid injections (TFESIs) have been shown in the past to be an effective treatment for low back pain with radicular symptoms [1-6]. Various corticosteroids have historically been used for these injections and are differentiated into two categories, particulate and non-particulate, based on their solubility in water as well as their tendency to aggregate [7]. Particulate corticosteroids tend to be larger in size $(0.5-100 \mu \mathrm{g})$ and less water soluble, resulting in possible aggregation and crystallization in hydrophilic environments. Particulate corticosteroids include methylprednisolone acetate, prednisolone acetate, triamcinolone acetonide, and betamethasone acetate. On the other hand, non-particulate corticosteroids like dexamethasone sodium phosphate tend to be smaller in size $(0.5 \mu \mathrm{g})$ and are freely water-soluble.

The safety of particulate corticosteroids for both cervical and lumbosacral epidural injections has been called into question since at least 2002, after the publication of multiple case reports and case series identifying severe post-injection complications including paraplegia, quadriplegia, stroke, and even death [8-15]. While the exact pathophysiology is still being investigated, the cause of these catastrophic complications is thought to be tied both to the larger size of the particulate molecules and their lower water solubility, both of which could lead to occlusion of smaller radiculomedullary arteries leading to the spinal cord $[16,17]$. The result of these reports culminated in safety announcements and a new label requirement by the U.S. Food and Drug Administration (FDA) in 2011 regarding epidural administration of triamcinolone acetonide. The documents state, "Epidural and intrathecal administration of this product is not recommended. Reports of serious medical events, including death, have been associated with epidural and intrathecal routes of corticosteroid administration... Spinal cord infarction, paraplegia, quadriplegia, cortical blindness, and stroke (including brainstem) have been reported after epidural administration of corticosteroids" [18].

Despite the concerns raised by the above cases and the FDA announcement, some practitioners still favor the use of particulate corticosteroids for thoracic and lumbar TFESIs. Some challenge the underlying embolization theories and attribute the complications to poor needle tip placement [19]. Others may support the continued use of particulate corticosteroids because of a few studies that suggest superior pain relief or duration of relief with particulate corticosteroids [20-22].

The purpose of this study was to compare the degree of pain relief between particulate and non-particulate lumbosacral TFESIs up to 6 months post-injection. While several studies have investigated this matter in the past, to our knowledge none have used a self-controlled design. By using this method many confounders have been eliminated. In our study, the same patient underwent a lumbosacral TFESI with each type of medication, at the same vertebral level and side. Our hypothesis was that there would be no significant difference in pain relief or duration of pain relief between the two types of corticosteroids. A secondary investigation of this study was to examine differences in functional improvement between the two types of medication, with the hypothesis that there also would be no significant difference.

\section{MATERIALS AND METHODS}

This was a retrospective, self-controlled study that was approved by the Institutional Review Board of the Medical College of Wisconsin (approval No. PRO00033474). The procedure log from a local pain medicine physician from $06 / 01 / 2009$ to $08 / 01 / 2018$ was reviewed. The charts of patients who had undergone more than one lumbosacral TFESI at the same level and side for the treatment of radicular low back pain at the level of injection were then reviewed. Further inclusion criteria for subjects in this study were 1) age greater than 18 years, 2) symptoms and physical exam findings suggestive of nerve root compression with confirmatory magnetic resonance imaging (MRI) findings, 3) a history of both types of corticosteroid injection at the same lumbosacral side and level, 4) no prior or subsequent spinal injection within 6 months, and 5) no subsequent spinal surgical intervention within the data collection period. Post-injection follow-up appointments were at 2, 3, and 6 months, at which time pain level and functional data were collected. The pain medicine physician involved in this study stopped using methylprednisolone for TFESIs and switched to dexamethasone in 2014 due to the safety concerns addressed above. Therefore, all of the methylprednisolone injections occurred first, and in some cases were years prior to the dexamethasone injections.

The primary outcomes for this study were both pain relief and duration of relief. Pain severity was measured by the visual analogue scale (VAS), which rates pain levels from $0-10$, with ' 0 ' meaning no pain and ' 10 ' meaning the worst possible pain. VAS data was collected at the appointment prior to the injection and at all subsequent followup appointments. A secondary outcome was functional improvement following injection, which was determined by a simple 'yes/no' question at each follow-up appointment regarding whether the patient believed their overall mobility or ability to perform activities of daily living's had 
improved since the TFESI.

\section{Procedure description}

Every injection was performed by the same boardcertified pain medicine physician. The traditional 'safe triangle' approach described in the well-known text by Berkwits et al. [23] was used for every subject. In brief, following local infiltration with $3 \mathrm{~mL}$ of $1 \%$ lidocaine hydrochloride, a 12.7-cm long, 22-gauge spinal needle was advanced towards the 6 o'clock position of the pedicle until bone was touched. The needle tip was then walked off inferiorly, and advanced until the 6 o'clock position of the pedicle was reached again in the anterior-posterior position. The fluoroscope was then redirected to the lateral position to verify needle placement. Negative aspiration was performed followed by injection of $1 \mathrm{~mL}$ of iohexol contrast medium. The imaging showed the spread of the contrast medium along the targeted nerve sheath with no intravascular, intrathecal, or subdural spread. After careful negative aspiration, a $3 \mathrm{~mL}$ mixture consisting of $2 \mathrm{~mL}$ $1 \%$ lidocaine hydrochloride and either $1 \mathrm{~mL} 40 \mathrm{mg}$ methylprednisolone acetate or $1 \mathrm{~mL} 10 \mathrm{mg}$ of dexamethasone sodium phosphate was injected. The needle tract was infiltrated with another $1 \mathrm{~mL}$ of $1 \%$ lidocaine hydrochloride while the needle was withdrawn.

\section{Statistical analysis}

Baseline comparisons between patient-specific characteristics (age, medications, and pre-injection VAS score) were completed with paired $t$-tests. Comparisons of VAS scores at specific follow-up visits were also performed with paired $t$-tests. Further, changes in VAS scores were analyzed using linear mixed models with a random subject effect and a random injection effect controlling for repeated measures. The effect of injection and its interaction with time on VAS changes were tested controlling for the effects of baseline VAS scores and time effect (follow-up visits) within linear mixed models. The use of the McNemar test for dichotomous variables, such as change in function, was evaluated, but this was eventually discarded due to the limited sample size created by the need for discordant pairs in order to run this analysis. Therefore, improvement in function is reported in percentages at each visit, along with $95 \%$ point-wise confidence intervals. The analysis was performed using R ver. 3.5.0 (R Foundation, Vienna, Austria). All continuous data were summarized by mean \pm standard deviation, and a $95 \%$ confidence interval (CI) was added where appropriate. $P$ values $<0.05$ were considered significant.
Table 1. Demographics and Baseline Pain Levels

\begin{tabular}{lccc}
\hline \multicolumn{1}{c}{ Variable } & $\begin{array}{c}\text { Methylpred- } \\
\text { nisolone }\end{array}$ & $\begin{array}{c}\text { Dexametha- } \\
\text { sone }\end{array}$ & $P$ value \\
\hline Age $(\mathrm{yr})$ & $63 \pm 16$ & $65 \pm 16$ & $<0.001$ \\
Sex (M/F) & $4 / 16$ & $4 / 16$ & $\mathrm{NA}$ \\
Duration of symptoms (yr) & $6 \pm 6$ & $8 \pm 6$ & 0.03 \\
Medications & & & \\
$\quad$ NSAIDs & 5 & 6 & 0.65 \\
$\quad$ Muscle relaxants & 12 & 9 & 1.0 \\
$\quad$ Neuromodulators & 13 & 10 & 0.25 \\
$\quad$ Opioids & $7.3 \pm 1.5$ & $6.8 \pm 2.4$ & 0.06 \\
Pre-injection pain level (VAS) & & 58 \\
\hline
\end{tabular}

Values are presented as mean \pm standard deviation or number only. NA: not applicable, NSAIDs: non-steroidal anti-inflammatory drugs, VAS: visual analogue scale (0: no pain, 10: maximal pain imaginable).

\section{RESULTS}

A total of 20 patients satisfied the inclusion criteria for this study. Demographic information is included in Table 1. The average age for the methylprednisolone group was 63 \pm 16 years vs. $65 \pm 16$ years for the dexamethasone group $(P<0.001)$. There were 16 females and 4 males. The mean duration of symptoms for the methylprednisolone group was $6 \pm 6$ years compared to $8 \pm 6$ years for dexamethasone $(P=0.03)$. The significant difference in age and duration of symptoms was anticipated, as the methylprednisolone injections all occurred prior to the dexamethasone TFESIs, as stated above. There were no differences in preinjection mean VAS scores between the groups (methylprednisolone mean VAS score $=7.3 \pm 1.5$; dexamethasone mean VAS score $=6.8 \pm 2.4 ; P=0.38$ ). The average duration between the two injections was $18.2 \pm 16.1$ months (range 6.3-69 mo). There was no significant difference in medications used prior to each injection (Table 1). Opioid use approached significance $(P=0.06)$, with three fewer patients prescribed opioids in the dexamethasone group; however, no medication had any significant impact on pain relief on further paired analysis. Etiologies of nerve root compression on MRI included central and paracentral disc herniations, often combined with some degree of facet arthropathy. The severity of foraminal stenosis included $2 / 20$ mild, $8 / 20$ moderate, and $10 / 20$ severe cases.

Fig. 1A portrays absolute values for the pre-injection and post-injection mean VAS scores. While the slope of the dexamethasone scores appears steeper after 2 months and crosses the methylprednisolone line at 6 months, these differences never reach a level of significance $(P>$ 0.05). Mean VAS scores were lower than baseline in both the methylprednisolone and dexamethasone groups at all three time points $(P<0.001)$.

Changes in mean VAS scores for each medication are reported in Fig. 1B. A decrease of $-3.4 \pm 3.0,-3.1 \pm 3.1$, and 

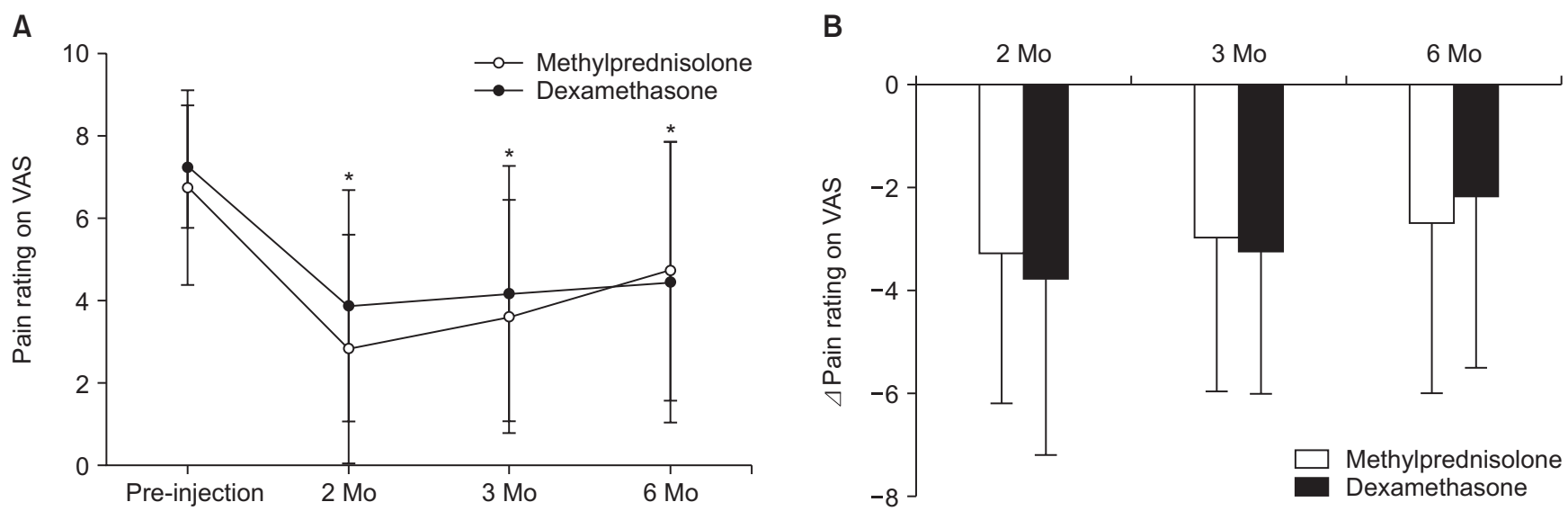

Fig. 1. Absolute pain rating (A) and change in pain rating (B) on the visual analogue scale (VAS) in patients receiving either methylprednisolone or dexamethasone lumbosacral transforaminal epidural steroid injections. Patients reported a significant reduction in pain at 2, 3, and 6 months compared to pre-injection values; however, there was no difference in pain reduction between the groups. Values are presented as mean \pm standard deviation. ${ }^{*} P<$ 0.05 vs. pre-injection.

$-2.8 \pm 3.4$ was seen for the methylprednisolone group at 2 , 3 , and 6 months, respectively. Similar decreases of $-3.9 \pm$ $3.5,-3.4 \pm 2.8$, and $-2.3 \pm 3.4$ were seen in the dexamethasone group. While all of these VAS scores were significantly improved compared to pre-injection scores $(P<0.001)$, there was no significant difference in pain relief at any point between the two types of medication.

Self-reported improvement in function for the two groups is presented in Fig. 2. The data is presented as the percentage of subjects in each group who reported improvement in function at each data collection point. For the methylprednisolone group, $65 \%, 51 \%$, and $41 \%$ endorsed improvement in function at 2, 3, and 6 months, respectively. For the dexamethasone group, the relevant values were $75 \%, 53 \%$, and $42 \%$.

\section{DISCUSSION}

The goal of this study was to investigate the difference in pain relief and functional improvement in patients who had undergone injections of both particulate and nonparticulate TFESIs. The primary results of this study show that there was no significant difference in the degree of pain relief for patients who underwent TFESI with each type of corticosteroid at the same lumbosacral level and side for up to 6 months. Our data also showed very similar rates of functional improvement for both types of medications at 2, 3, and 6 months.

Similar results between particulate and non-particulate corticosteroids have been reported in the literature. Denis et al. [24] compared the efficacy of $7.5 \mathrm{mg}$ dexamethasone to $6 \mathrm{mg}$ betamethasone in a double-blind, randomized control trial (RCT) $(n=56)$. They followed patients at 1-, 3-,

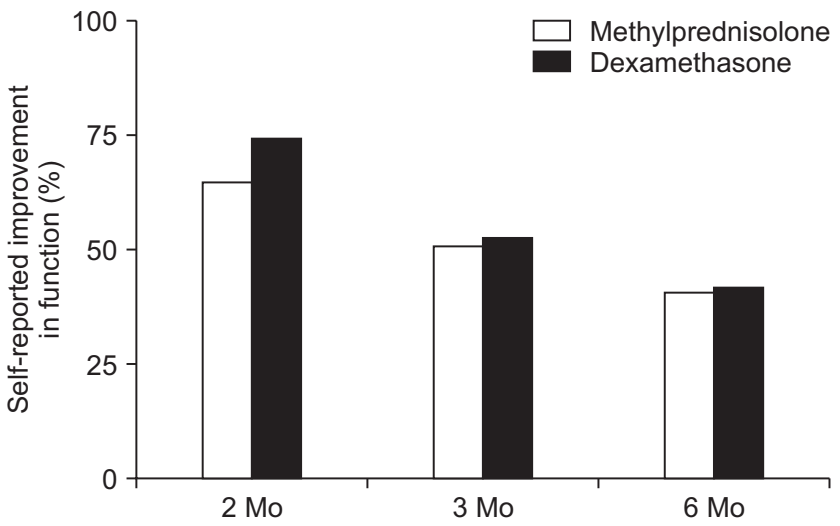

Fig. 2. Self-reported improvement in function in patients receiving either methylprednisolone or dexamethasone lumbosacral transforaminal epidural steroid injections. Values are presented as percentage of respondents who self-reported improvement in function.

and 6-month intervals with change in VAS score as a primary outcome, and functional improvement as measured by the Oswestry disability index (ODI) as a secondary outcome. A significant decrease in VAS scores compared to pre-injection levels was noted in both groups at 3 and 6 months, as was the decrease in ODI scores. However, no significant difference was found between the groups for difference in VAS scores. A multivariate regression analysis did show statistically significant improvement in ODI scores for the dexamethasone group at the 6-month interval compared to the betamethasone group, whereas our study showed similar rates of functional improvement at all data points.

Kennedy et al. [25] performed a double-blind RCT with 78 subjects who were divided into groups who underwent single-level TFESIs either with $15 \mathrm{mg}$ dexamethasone or $60 \mathrm{mg}$ triamcinolone acetonide. They found no signifi- 
cant difference in pain relief between the two groups at 2 weeks, 3 months, and 6 months. They also found comparable rates of eventual spinal surgery $(14.6 \%$ for the dexamethasone group and $18.9 \%$ for the triamcinolone). The dexamethasone group, however, did have a significantly higher percentage of patients undergo 3 injections compared to the triamcinolone group $(17.1 \%$ and $2.7 \%$, respectively $[P=0.0052])$, which may have impacted results.

In contrast, other studies have shown significant differences between the effects of particulate and nonparticulate TFESIs. Park et al. [22] performed an RCT with a sample of 106 subjects who were divided into groups who received either $40 \mathrm{mg}$ triamcinolone or $7.5 \mathrm{mg}$ dexamethasone. Primary outcomes included the decrease in pain as measured by the VAS, and the short-form McGill pain questionnaire, as well as improvement in function per the revised ODI. Post-injection data were collected at one follow-up visit at 4 weeks. While this study showed significant improvement in pain scores compared to the preinjection scores for both groups, the particulate corticosteroid group had a significantly greater reduction in pain compared to the non-particulate group on the VAS score (-5.9 for triamcinolone vs. -3.3 for dexamethasone). However, no significant difference was found for the McGill pain questionnaire nor the revised ODI. It is worth noting, the Park et al.'s study [22] also differs from this study by using a lower dose of $7.5 \mathrm{mg}$ dexamethasone.

El-Yahchouchi et al. [26] performed a retrospective study of 2,634 radicular pain subjects and compared the results of three different corticosteroid TFESIs: $10 \mathrm{mg}$ dexamethasone, $80 \mathrm{mg}$ triamcinolone, and $12 \mathrm{mg}$ betamethasone. Primary outcomes included changes in pain level according to the numerical rating scale (NRS), as well as functional disability according to the Roland-Morris disability questionnaire (R-M). The authors evaluated both continuous scores as well as categorical outcomes. They defined successful pain relief as $>50 \%$ reduction in NRS or a report of $0 / 10$ pain. A successful functional response was a decrease in R-M score by $40 \%$ or more. When analyzed categorically, there was no significant difference in either pain relief or function between the three corticosteroids at 2 weeks and 2 months post-injection. However, the continuous outcomes did reveal the superiority of dexamethasone at 2 months post-injection in both pain relief and functional improvement $(-0.57$ [CI -0.89 to -0.31 ] for change in NRS, -1.1 [CI -1.7 to -0.5$]$ for change in R-M). It was noted that while this was a statistically significant difference, the absolute values involved ( -0.57 on NRS and -1.1 on R-M) are small and the clinical significance may be questioned.

As noted above, the current literature comparing the effectiveness of particulate and non-particulate lumbosacral TFESIs has shown varying results. While some studies may show subtle differences in pain relief, duration of relief, or functional improvement at one moment or another, it is important to note that these studies all show significant improvement in pain levels for weeks to months after injection compared to baseline levels of pain. The study presented here showed no statistical difference in pain relief or duration of relief up to 6 months post-injection between $10 \mathrm{mg}$ dexamethasone and $40 \mathrm{mg}$ methylprednisolone TFESIs when administered sequentially to the same patients. Given the fact that there are serious safety concerns for the particulate corticosteroid injections, the findings of this study would strongly support the use of non-particulate corticosteroids for lumbosacral TFESIs. We believe that the value and novelty of this study is that it was self-controlled: the same subject underwent TFESIs with each type of medication at the same level and side. To our knowledge, the results of such a strict study design and the inherent control of variables has not been reported in the literature.

This study did have limitations. First, it was a retrospective study and therefore bears the increased risk of selection bias inherent in such study designs. The small sample is another limitation, as only 20 subjects were found to satisfy the very strict inclusion criteria. However, given how closely pain reduction and improvement in function were matched between the groups it is unlikely having a higher number of patients would yield different results than those presented here. As noted above, no statistical analysis was able to be performed regarding improvement in function. A paired analysis of these data is only possible with discordant results, and rate of similar results yielded an insufficient sample for analysis.

This study demonstrates the similar efficacy in pain relief and duration of relief up to 6 months between $10 \mathrm{mg}$ dexamethasone and $40 \mathrm{mg}$ methylprednisolone lumbosacral TFESIs for the treatment of lumbosacral radicular pain. Our results also suggest similar rates of functional improvement between the two corticosteroid injections at 2,3 , and 6 months. These findings support the use of nonparticulate corticosteroids for lumbosacral TFESIs in the context of severe safety risks with particulate corticosteroids.

\section{CONFLICT OF INTEREST}

No potential conflict of interest relevant to this article was reported. 


\section{FUNDING}

No funding to declare.

\section{ORCID}

Nicholas K. Donohue, https://orcid.org/0000-0001-6280-7158

Sergey S. Tarima, https://orcid.org/0000-0001-7542-1094

Matthew J. Durand, https://orcid.org/0000-0002-1416-1652

Hong Wu, https://orcid.org/0000-0003-1470-2794

\section{REFERENCES}

1. Buenaventura RM, Datta S, Abdi S, Smith HS. Systematic review of therapeutic lumbar transforaminal epidural steroid injections. Pain Physician 2009; 12: 233-51.

2. Ghahreman A, Ferch R, Bogduk N. The efficacy of transforaminal injection of steroids for the treatment of lumbar radicular pain. Pain Med 2010; 11: 1149-68.

3. Kaufmann TJ, Geske JR, Murthy NS, Thielen KR, Morris JM, Wald JT, et al. Clinical effectiveness of single lumbar transforaminal epidural steroid injections. Pain Med 2013; 14: 112633.

4. MacVicar J, King W, Landers MH, Bogduk N. The effectiveness of lumbar transforaminal injection of steroids: a comprehensive review with systematic analysis of the published data. Pain Med 2013; 14: 14-28.

5. Roberts ST, Willick SE, Rho ME, Rittenberg JD. Efficacy of lumbosacral transforaminal epidural steroid injections: a systematic review. PM R 2009; 1: 657-68.

6. Vad VB, Bhat AL, Lutz GE, Cammisa F. Transforaminal epidural steroid injections in lumbosacral radiculopathy: a prospective randomized study. Spine (Phila Pa 1976) 2002; 27: 11-6.

7. Dietrich TJ, Sutter R, Froehlich JM, Pfirrmann CW. Particulate versus non-particulate steroids for lumbar transforaminal or interlaminar epidural steroid injections: an update. Skeletal Radiol 2015; 44: 149-55.

8. Hodler J, Boos N, Schubert M. Must we discontinue selective cervical nerve root blocks? Report of two cases and review of the literature. Eur Spine J 2013; 22 Suppl 3: S466-70.

9. Houten JK, Errico TJ. Paraplegia after lumbosacral nerve root block: report of three cases. Spine J 2002; 2: 70-5.

10. Huntoon MA, Martin DP. Paralysis after transforaminal epidural injection and previous spinal surgery. Reg Anesth Pain Med 2004; 29: 494-5.

11. Kennedy DJ, Dreyfuss P, Aprill CN, Bogduk N. Paraplegia following image-guided transforaminal lumbar spine epidural steroid injection: two case reports. Pain Med 2009; 10: 138994.
12. Ludwig MA, Burns SP. Spinal cord infarction following cervical transforaminal epidural injection: a case report. Spine (Phila Pa 1976) 2005; 30: E266-8.

13. Popescu A, Lai D, Lu A, Gardner K. Stroke following epidural injections--case report and review of literature. J Neuroimaging 2013; 23: 118-21.

14. Somayaji HS, Saifuddin A, Casey AT, Briggs TW. Spinal cord infarction following therapeutic computed tomographyguided left L2 nerve root injection. Spine (Phila Pa 1976) 2005; 30: E106-8.

15. Wybier M, Gaudart S, Petrover D, Houdart E, Laredo JD. Paraplegia complicating selective steroid injections of the lumbar spine. Report of five cases and review of the literature. Eur Radiol 2010; 20: 181-9.

16. Derby R, Lee SH, Date ES, Lee JH, Lee CH. Size and aggregation of corticosteroids used for epidural injections. Pain Med 2008; 9: 227-34.

17. MacMahon PJ, Shelly MJ, Scholz D, Eustace SJ, Kavanagh EC. Injectable corticosteroid preparations: an embolic risk assessment by static and dynamic microscopic analysis. AJNR Am J Neuroradiol 2011; 32: 1830-5.

18. Food and Drug Administration. Kenalog ${ }^{\circledR}-10$ injection (triamcinolone acetonide injectable suspension, USP) [Internet]. Silver Spring (MD): Food and Drug Administration; 2011. Available at: https://www.accessdata.fda.gov/drugsatfda docs/label/2011/012041s038lbl.pdf.

19. Shah RV. Paraplegia following thoracic and lumbar transforaminal epidural steroid injections: how relevant are particulate steroids? Pain Pract 2014; 14: 297-300.

20. Dreyfuss P, Baker R, Bogduk N. Comparative effectiveness of cervical transforaminal injections with particulate and nonparticulate corticosteroid preparations for cervical radicular pain. Pain Med 2006; 7: 237-42.

21. Lee JW, Park KW, Chung SK, Yeom JS, Kim KJ, Kim HJ, et al. Cervical transforaminal epidural steroid injection for the management of cervical radiculopathy: a comparative study of particulate versus non-particulate steroids. Skeletal Radiol 2009; 38: 1077-82.

22. Park CH, Lee SH, Kim BI. Comparison of the effectiveness of lumbar transforaminal epidural injection with particulate and nonparticulate corticosteroids in lumbar radiating pain. Pain Med 2010; 11: 1654-8.

23. Berkwits L, Davidoff SJ, Buttaci CJ, Furman MB. Lumbar transforaminal epidural steroid injection, supraneural (traditional) approach. In: Atlas of image-guided spinal procedures. Edited by Furman MB, Lee TS, Berkwits L. Philadelphia, Elsevier Saunders. 2012, pp 93-103.

24. Denis I, Claveau G, Filiatrault M, Fugère F, Fortin L. Randomized double-blind controlled trial comparing the effectiveness of lumbar transforaminal epidural injections of particulate and nonparticulate corticosteroids for lumbosacral radicular pain. Pain Med 2015; 16: 1697-708. 
25. Kennedy DJ, Plastaras C, Casey E, Visco CJ, Rittenberg JD, Conrad B, et al. Comparative effectiveness of lumbar transforaminal epidural steroid injections with particulate versus nonparticulate corticosteroids for lumbar radicular pain due to intervertebral disc herniation: a prospective, randomized, double-blind trial. Pain Med 2014; 15: 548-55.
26. El-Yahchouchi C, Geske JR, Carter RE, Diehn FE, Wald JT, Murthy NS, et al. The noninferiority of the nonparticulate steroid dexamethasone vs the particulate steroids betamethasone and triamcinolone in lumbar transforaminal epidural steroid injections. Pain Med 2013; 14: 1650-7. 